\title{
Understanding the role of gut microbiome in metabolic disease risk
}

\author{
Yolanda Sanz ${ }^{1}$, Marta Olivares ${ }^{1}$, Ángela Moya-Pérez¹ and Carlo Agostoni²
}

The gut microbiota structure, dynamics, and function result from interactions with environmental and host factors, which jointly influence the communication between the gut and peripheral tissues, thereby contributing to health programming and disease risk. Incidence of both type-1 and type-2 diabetes has increased during the past decades, suggesting that there have been changes in the interactions between predisposing genetic and environmental factors. Animal studies show that gut microbiota and its genome (microbiome) influence alterations in energy balance (increased energy harvest) and immunity (inflammation and autoimmunity), leading to metabolic dysfunction (e.g., insulin resistance and deficiency). Thus, although they have different origins, both disorders are linked by the association of the gut microbiota with the immune-metabolic axis. Human studies have also revealed shifts in microbiome signatures in diseased subjects as compared with controls, and a few of them precede the development of these disorders. These studies contribute to pinpointing specific microbiome components and functions (e.g., butyrate-producing bacteria) that can protect against both disorders. These could exert protective roles by strengthening gut barrier function and regulating inflammation, as alterations in these are a pathophysiological feature of both disorders, constituting common targets for future preventive approaches.

$\mathbf{T}$ he bacterial ecosystem (microbiota) living in our intestine plays a fundamental role in the normal functioning of both metabolic and immune systems, beyond genetic determinants. Consequently, imbalances in gut microbiota (dysbiosis) are a possible causal factor of metabolic and autoimmune diseases. The initial colonization of the newborn intestine is particularly relevant to the proper development of the host's immune and metabolic functions and to determine disease risk in early and later life (1). Gut microbiota signatures seem to be highly specific for each individual, resulting in large interindividual variations that depend on both host genetics and environmental factors $(1,2)$. This specificity has hindered our understanding of the microbiota's role in health and disease. However, the fact that gut microbiota shows certain plasticity, particularly in response to the diet, also makes it possible to develop intervention strategies that promote a healthy gut ecosystem to reduce disease risk (3). Breast milk-associated bifidobacteria in infants are a hallmark of the host-gut microbiome response to diet, promoting a particular microbial community structure, and presumably contributing to the role of breastfeeding in reducing disease risk (e.g., infections, obesity, and type- 2 diabetes) (4). Although microbiota seems to be more sensitive to environmental factors in infants than in adults, the latter also respond to dietary intervention with variations of at least $10 \%$ (2). There is also evidence that gut microbiota and their products have large and diverse effects on immunity and immunemediated disorders (5). Furthermore, bacterial interactions (mutualism and antagonism) that define the gut ecosystem are known to be indirectly mediated by the host immune system and particularly by its innate components $(6,7)$. These findings reveal the two-way communication between the gut microbiota and the host, which might be shifted by diet and influence the risk of developing immune-mediated disorders, particularly at early stages of development.

In the light of this evidence, the increased incidence of dietassociated inflammatory diseases (obesity and type- 2 diabetes) and autoimmune disorders (e.g., type-1 diabetes) could be partially explained by changes in gene-environment interactions, including diet-induced changes in microbiota. Systemic inflammation and autoimmunity are detectable years prior to the onset of overt disease, promoting the development of metabolic and autoimmune diseases (8). This suggests that subjects predisposed to disease might benefit from interventions targeting the immune system directly or indirectly before the disease manifests. Thus, new biomarkers of disease progression, including those related to the microbiome, must be identified to discover the pathophysiological determinants of these disorders and develop preventive interventions in the form of personalized health-care and nutrition-based strategies.

Here, we will consider two diseases, type- 1 and type- 2 diabetes, as both disorders are linked to the gut microbiota association with the immune-metabolic axis (Figure 1). We will provide a comprehensive review of the extent to which

${ }^{1}$ Microbial Ecology, Nutrition \& Health Research Group, Institute of Agrochemistry and Food Technology, National Research Council (IATA-CSIC), Valencia, Spain; ${ }^{2}$ Pediatric Clinic, Department of Clinical Sciences and Community Health, University of Milan, Fondazione IRCCS Ca' Granda-Ospedale Maggiore Policlinico, Milan, Italy. Correspondence: Yolanda Sanz (yolsanz@iata.csic.es) 


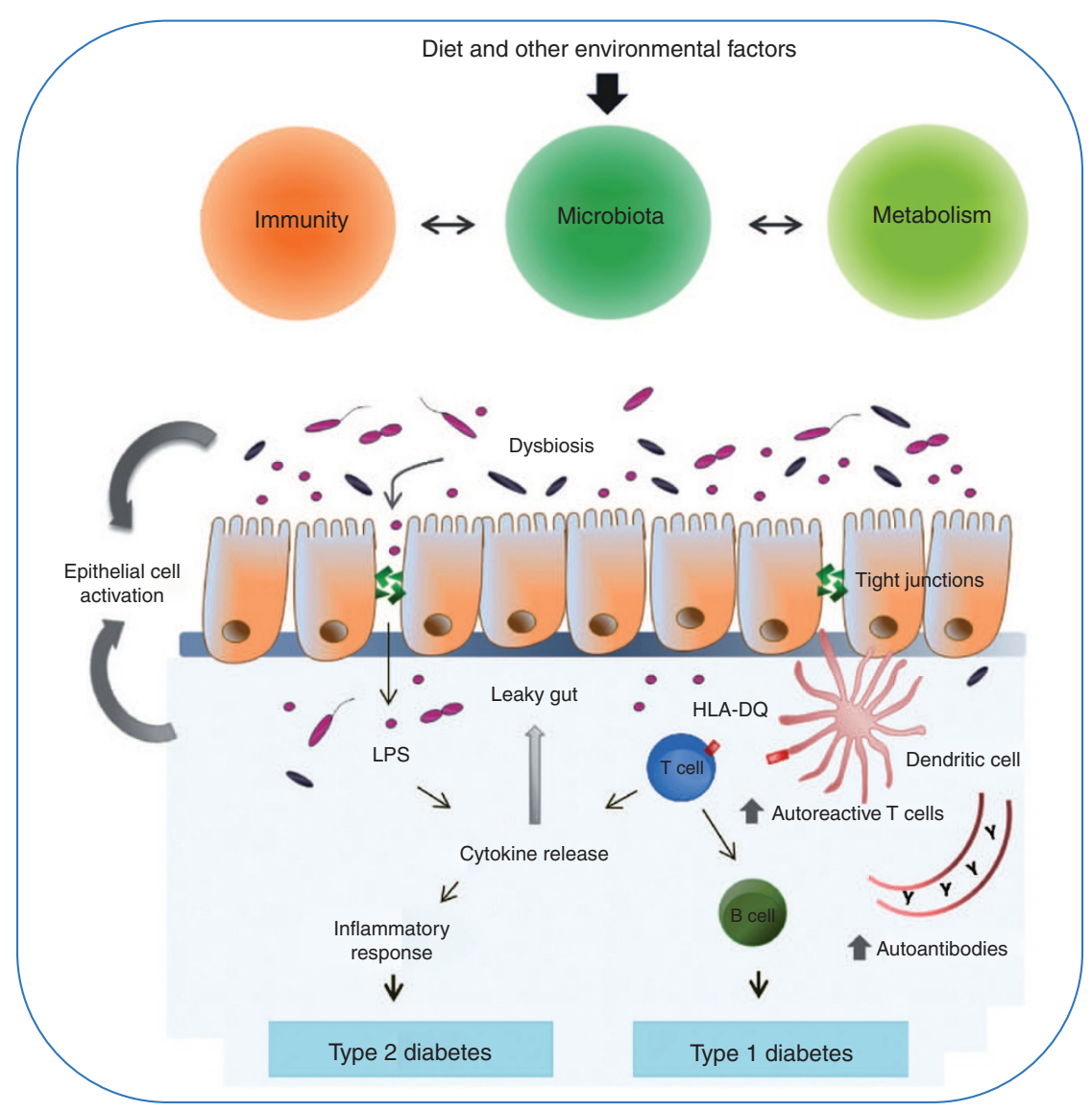

Figure 1. Schematic representation of the interaction of the gut microbiota with the immune-metabolic axis and the different mechanisms proposed to explain its implication in health and disease risk. In type-1 diabetes, the intestinal dysbiosis and the increased gut permeability and altered immunoregulatory mechanisms seem to trigger the autoimmune response leading to the destruction of $\beta$ cells in the pancreatic islets. In type- 2 diabetes, saturated fat and dysbiosis due to "obesogenic" diets cause inflammation and alterations in gut permeability contributing to disease onset.

available evidence supports a role for gut microbiota composition and function in disease risk. We will also identify the gaps in the understanding of the respective roles of gut microbiota, dietary habits, and host factors in disease progression and prevention.

\section{GUT MICROBIOTA AND RISK OF DEVELOPING OBESITY AND TYPE-2 DIABETES}

Obesity results from a positive imbalance between energy intake and expenditure and is also associated with low-grade inflammation leading to chronic metabolic disease (type-2 diabetes). The steady increase in obesity prevalence has mainly been attributed to socio-economic factors, dietary changes, and sedentary lifestyle. Nevertheless, recent evidence suggests that our gut microbiota and microbiome also contribute to the host genetic make-up and to the whole metabolic activity of the body and immune function, and therefore, could play a role in these disorders.

\section{Mechanisms Behind the Role of Gut Microbiota in Obesity and Type-2 Diabetes in Animals}

Alterations in gut microbiota structure and function detected in both genetically and diet-induced obesity models led to initially establishing a role of microbiota in body-weight regulation (9). Nevertheless, gut microbiota alterations associated with obesity were also proposed to be a secondary consequence of the diets inducing obesity or of the genotype (6). However, comparison of germ-free and conventionally colonized mice has demonstrated that gut microbiota colonization leads to impaired glucose metabolism and increased macrophage accumulation in white adipose tissue not only in mice fed a high-fat diet but also fed a standard diet, suggesting that microbiota effects are partly independent of diet (10). Also, animals with a common genotype and fed the same diet can develop different metabolic phenotypes (either diabetic or nondiabetic) as a function of their specific gut microbiota profile, suggesting that gut microbiota per se determines the risk of developing metabolic dysfunction to some extent (11).

Figure 2 provides a summary of evidence for the possible mode of action of the microbiota in obesity and the associated comorbidities, including type- 2 diabetes. Initially, comparisons between germ-free and conventional colonized mice indicated that the microbiota, as a whole, increases our ability to extract energy from the diet and store this energy in peripheral tissues (liver, adipose tissue, etc.), leading to impairment of insulin sensitivity (12). The fact the microbiota breaks down indigestible dietary components (mainly plant-derived polysaccharides) is considered one of the mechanisms improving 


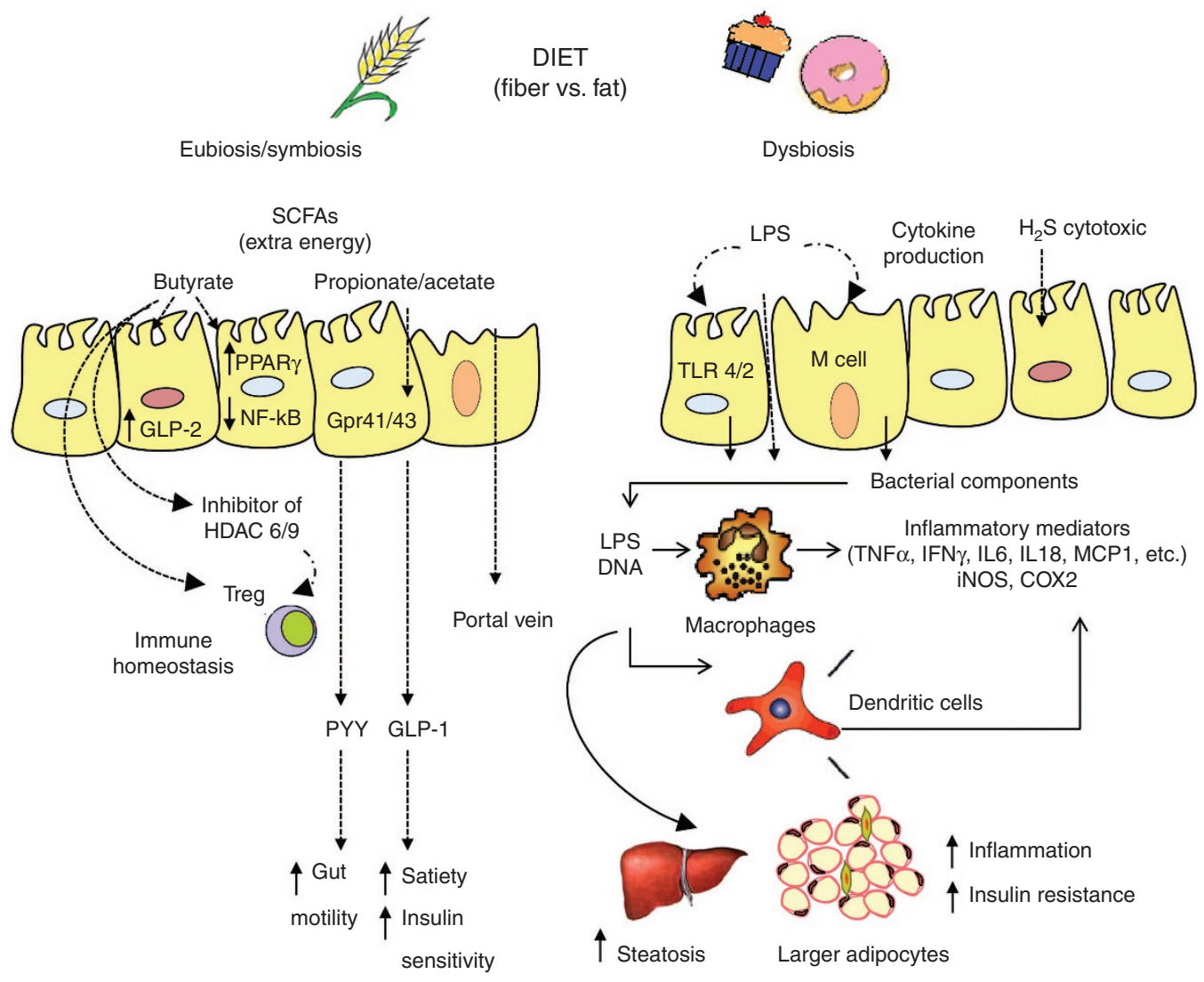

Figure 2. Main mechanisms of action of gut microbiota and derived metabolites in obesity and associated metabolic dysfunctions (insulin resistance and type-2 diabetes). Gut microbiota contributes to the hydrolysis of complex polysaccharides from dietary fiber, and thereby might contribute to increasing energy harvest and to generating short-chain fatty acids (SCFAs; acetic propionic and butyric acid) that affect the host's metabolism in different ways. SCFAs might activate the G-protein coupled receptor (Gpr) 41 inducing the expression of peptide YY, an intestinal hormone that influences gut motility, increases intestinal transit rate, and reduces energy harvest from the diet. SCFAs might also activate Gpr43 and Gpr41 inducing glucagon-like peptide-1 (GLP-1) secretion, increasing insulin sensitivity, and inducing satiety. Butyrate provides energy to enterocytes, exerting a trophic effect and inducing the synthesis of GLP-2, thereby strengthening the gut barrier function. Butyrate may also promote the formation of peripheral regulatory $T$ cells (Treg) by its ability to inhibit the histone deacetylases 6 and 9 that leads to acetylation of histone H3, which promotes the expression of the Treg-specific forkhead transcription factor FoxP3. Intestinal dysbiosis, which could be partly caused by "obesogenic" diets rich in saturated fats, may lead to the growth of potential pathogens (Gram-negative bacteria and derived lipopolysaccharide (LPS)) with proinflammatory effects via generation of cytotoxic compounds $\left(\mathrm{H}_{2} \mathrm{~S}\right)$ or interaction with innate immune receptors (TLR4, TLR2) and contribute to inflammatory cytokine production, attraction of inflammatory cells, and translocation of bacterial products (LPS, DNA) by transcellular and paracellular pathways that activate inflammation in peripheral tissues. NF, nuclear factor.

the host's ability to extract energy from the diet. This is consistent with evidence that the microbiome of human feces is enriched in genes involved in the utilization of complex dietary polysaccharides (13). This activity leads to the generation of short-chain fatty acids (SCFAs; butyric, acetic, and propionic) and gases (e.g., hydrogen), which can also be further metabolized, activating the overall colonic fermentation and increasing the efficiency of energy extraction from ingested nutrients (14). Although the energy contribution of complex plant polysaccharides is by far lower than that produced by digestible carbohydrates, their metabolism can constitute a survival strategy mediated by our symbiotic microbiota. However, the role of the gut microbiota in supplying extra energy from the diet could be less prominent in the context of Western diets rich in fats and simple carbohydrates. This theory of "increased energy harvest" is somehow contradictory to the benefits on metabolic health attributed to high dietary fiber intake and the SCFAs generated. Some of the SCFAs resulting from intake of high fiber diets, and particularly butyrate, are considered to exert beneficial effects in the context of obesity and associated comorbidities. One of the proposed modes of action of SCFAs (specially of butyrate) is related to their ability to increase satiety and decrease calorie intake and postprandial glycemia via modification of gut peptide production (glucagon-like peptide (GLP)-1 and gastric inhibitory peptide). In addition, butyrate is the main energy source for enterocytes, and therefore, regulates cell proliferation and differentiation and induces GLP-2 production, which altogether strengthens the gut barrier function. Butyrate also reduces oxidative damage and inflammation by inhibiting histone deacetylases and the activation of the transcription factor nuclear factor- $\mathrm{\kappa B}$ and the associated cytokine production (15,16; Figure 2).

Inflammation seems to be one of the major pathophysiological factors leading to insulin resistance and progressively to type-2 diabetes. Gut microbiota alterations can also contribute to this inflammatory condition. Obesogenic diets may promote the growth of pathobionts (potential pathogens), which could trigger an inflammatory response via local 


\section{Gut microbiome and metabolic disease}

activation of innate immune receptors (e.g., TLR4, TLR2) with production of proinflammatory cytokines $(17,18)$ or via production of toxic compounds (e.g., hydrogen sulphide) (19). Both mechanisms could also lead to a leaky gut, translocation of microbial molecules, and overall, promote systemic inflammation. Also, saturated fatty acids of obesogenic diets might favor a leaky gut by inducing expression and activation of innate immune receptors (Toll-like receptors) and increasing lymphocyte flux and proliferation; altogether this may lead to the production of cytokines (e.g., tumor necrosis factor-alpha, interferon-gamma) that increase intestinal permeability, facilitating translocation of bacterial products regardless of possible alterations in the gut microbiota composition. Lipopolysaccharide can also translocate via a transcellular epithelial pathway together with chylomicrons formed to incorporate dietary long-chain fatty acids in the form of triglycerides or through intestinal-epithelial microfold (M) cells (9). Murine models of high-fat diet-induced obesity have also demonstrated that live Gram-negative commensal intestinal bacteria (Escherichia coli) can translocate to the blood and adipose tissue and that this process is mediated by dendritic cells and depends on innate immunity pattern-recognition receptors (TLR4 and Nod1) (20). However, intervention in the gut ecosystem with specific prebiotics or potential probiotics reduces the high-fat diet-induced metabolic endotoxemia, inflammatory tone, bacterial product translocation, and metabolic dysfunction, demonstrating that these effects are partly mediated by gut microbiotainduced changes in animal models $(20,21)$.

\section{Evidence Supporting a Role of Gut Microbiota in Obesity in Humans}

In humans, a large number of studies have established relationships between alterations in gut microbiota structure and function, and obesity, although there is no consensus on the key players in this disorder yet. Numerous studies report reduced proportions of Bacteroidetes or its subgroups (e.g., Bacteroides) in obese subjects compared with lean subjects, parallel to increased proportions of Firmicutes or its subgroups $(22,23)$. However, other studies have not found consistent results $(22,24)$. These include a recent metagenomic study reporting that subjects with low bacterial richness (low gene count) gained more weight and had increased inflammatory tone ( $\mathrm{C}$ reactive protein and leptin), insulin resistance and dyslipidemia as compared with subject with high bacterial gene counts (24). This finding supports the idea that a less diverse microbiota is less resilient to the invasion of unhealthy microbes, which may contribute to disease. Bacteroides and Ruminococcus spp. were more abundant in subjects with low gene counts and metabolic dysfunction, whereas Faecalibacterium prausnitzii, Bifidobacterium, Lactobacillus, Alistipes, Akkermansia, among others, were more dominant in subjects with high gene counts and healthier metabolic phenotypes. Nonetheless, not all subjects with either a high or low gene counts have a similar metabolic phenotype, suggesting that findings could be influenced by confounding factors.
Proteobacteria or enterobacteria were positively associated with obesity in a few studies, including one in preschool children, as well as Staphylococcus spp. in pregnant women (22-24). By contrast, Akkermansia spp. was associated with a lean phenotype in two human studies, one conducted in children (22).

Diet is a major driver of gut microbiota composition, which may partly explain why microbiota alterations are associated with an obese phenotype in humans. However, the microbiota structure resulting from unhealthy dietary habits is also thought to contribute to body-weight regulation (25). In this context, higher energy intake leading to an increased ratio of Firmicutes to Bacteroidetes has also been related to increased energy absorption from diet (25). Human intervention studies have also revealed that the individual's microbiota composition and richness could influence the effectiveness of dietary interventions in improving the metabolic phenotype $(26,27)$. Furthermore, transplantation experiments of human microbiota in mouse models reveal that the influence of dietmicrobiota interactions on the host metabolic phenotype is transmissible. A recent study has shown that when microbiota from subjects discordant for obesity (uncultured fecal communities and the corresponding fecal bacterial cultures) is transplanted in germ-free mice, these mice develop the corresponding phenotype when fed a low in fat and high in plant polysaccharides chow diet (28). The same study showed cohousing mice harboring the obese human microbiota with mice containing the lean human microbiota prevented the development of an obesity-associated metabolic phenotype due to the invasion of the obese mouse intestine with specific members of Bacteroidetes (e.g., Bacteroides uniformis, B. caccae, B. cellulosilyticus, etc.) from the lean microbiota. However, this effect was diet-dependent and positive only in the context of a chow diet representing low saturated fat, high fruit, and vegetable intake, but not in the context of a chow diet, representing high saturated fat, low fruit, and vegetable intake (28). These findings were also supported by administration of a selected Bacteroides strain (B. uniformis CECT 7771) from humans to a mouse model of diet-induced obesity in an independent study (29). Furthermore, recent fecal transplants to type-2 diabetic subjects beneficially influenced glucose metabolism and insulin resistance, proving that microbiota replacement strategies could help to protect from metabolic disease in humans (30).

Despite all this evidence, prospective epidemiological studies are necessary to establish whether specific microbiota features constitute risk factors and predict obesity and the associated metabolic disorders; however, only a couple of studies have been published to date. A follow-up study including 49 infants has reported that differences in fecal microbiota composition at 6 and 12 mo of age precede subsequent overweight in children at $7 \mathrm{y}$ of age. Children maintaining normal weight showed a greater number of bifidobacteria, while children who became overweight harbored a greater number of Staphylococcus aureus during infancy (31). A more recent and larger study of 330 healthy Danish infants from 9 to 36 mo of age reported a positive correlation between the increase in BMI 
and the increase in SCFA-producing clostridia (the Clostridum leptum group and Eubacterium hallii) (32). Taking into consideration the positive associations established between rapid infant weight gain and later-life obesity, these microbiological differences could play a role in obesity; however, direct evidence must be established. Santacruz et al. (33) detected positive correlations between mother's $E$. coli numbers and birth body weight, but data for the effects on offspring were not followed-up. Another study concluded that microbiota in 79 infants born large for gestational age differed significantly compared with microbiota in infants born with an average weight for gestational age. Gram-negative Proteobacteria were more abundant in neonates born large for gestational age, whereas Gram-positive Firmicutes were more abundant in neonates born average for gestational age (34). Due to the fact that high birth weight is a risk factor for the development of metabolic disorders, these findings suggest that early microbiota could be a factor programming immune and metabolic health, but data from follow-up studies were not reported.

\section{Evidence Supporting the Role of Gut Microbiota in Type-2 Diabetes in Humans}

Studies reporting associations between shifts in gut microbiota composition and function and type- 2 diabetes in humans are summarized in Table 1. Some of the findings suggest that metabolic markers of disease could be relevant for defining the relationships between obesity and gut microbiota (35). A recent metagenomic study also led to the development of a mathematical model to identify metagenomic markers for type- 2 diabetes and diabetes-like metabolism (38). Nevertheless, the discriminant metagenomic markers were shown to differ between different cohorts of subjects, revealing that our understanding is insufficient to enable prediction of an individual's disease risk based solely on the gut microbiome. Notwithstanding, a decreased abundance of some butyrate-producing bacteria (e.g., Roseburia, F. prausnitzii) and an increased abundance of opportunistic pathogens (e.g., Clostridium clostridioforme, E. coli) could be a potential microbiome signature of reduced glucose tolerance and type-2 diabetes. However, prospective

Table 1. Changes in the intestinal microbiota associated with type-2 diabetes in human

\begin{tabular}{|c|c|c|c|c|}
\hline \multirow[b]{2}{*}{ Study group } & \multirow[b]{2}{*}{ Methodology } & \multicolumn{2}{|c|}{ Microbiota change $^{\mathrm{a}}$} & \multirow[b]{2}{*}{ Reference } \\
\hline & & Phylum or class & Genus or species & \\
\hline \multirow{2}{*}{$\begin{array}{l}\text { Diabetics vs. nondiabetics } \\
(n=18 \text { vs. } n=18)\end{array}$} & \multirow[t]{2}{*}{ Real-time PCR } & 个Betaproteobacteria & & \multirow[t]{2}{*}{35} \\
\hline & & $\downarrow$ Firmicutes (Clostridia) & & \\
\hline \multirow{2}{*}{$\begin{array}{l}\text { Diabetics vs. nondiabetics } \\
(n=16 \text { vs. } n=12)\end{array}$} & \multirow[t]{2}{*}{ Real-time PCR } & & $\downarrow$ Bifidobacterium & \multirow[t]{2}{*}{36} \\
\hline & & & $\downarrow$ Bacteroides vulgatus & \\
\hline \multirow{13}{*}{$\begin{array}{l}\text { Diabetics vs. nondiabetic } \\
(n=71 \text { vs. } n=74)\end{array}$} & \multirow[t]{13}{*}{ Shotgun sequencing } & & $\uparrow$ Bacteroides caccae & \\
\hline & & & 个Clostridium hathewayi & \\
\hline & & & $\uparrow C l o s t r i d i u m$ ramosum & \\
\hline & & & $\uparrow$ Clostridium symbiosum & \\
\hline & & & 个Eggerthella lenta & \\
\hline & & & $\uparrow$ Escherichia coli & 37 \\
\hline & & & 个Akkermansia muciniphila & \\
\hline & & & 个Desulfovibrio & \\
\hline & & & $\downarrow$ Clostridiales sp. SS3/4 & \\
\hline & & & $\downarrow$ Eubacterium rectal & \\
\hline & & & $\downarrow$ Faecalibacterium prausnitzii & \\
\hline & & & $\downarrow$ Roseburia intestinalis & \\
\hline & & & $\downarrow$ Roseburia inulinivorans & \\
\hline \multirow{4}{*}{$\begin{array}{l}\text { Diabetics vs. nondiabetics } \\
(n=53 \text { vs. } n=43)\end{array}$} & \multirow[t]{4}{*}{ Shotgun sequencing } & & 个Lactobacillus spp. & \multirow[t]{4}{*}{38} \\
\hline & & & $\downarrow$ Clostridium spp. & \\
\hline & & & $\uparrow$ Clostridium clostridioforme & \\
\hline & & & $\downarrow$ Roseburia & \\
\hline Prediabetics vs. nondiabetics & \multirow[t]{2}{*}{$16 \mathrm{~S}$ rDNA sequencing } & & $\downarrow$ Akkermansia muciniphila & \multirow[t]{2}{*}{39} \\
\hline$(n=64$ vs. $n=44)$ & & & $\downarrow$ Faecalibacterium prausnitzii & \\
\hline $\begin{array}{l}\text { Diabetics vs. prediabetics } \\
\text { ( } n=13 \text { vs. } n=64)\end{array}$ & 16S rDNA sequencing & & $\downarrow$ Bacteroides & 39 \\
\hline
\end{tabular}




\section{Gut microbiome and metabolic disease}

epidemiological studies in well-controlled populations are needed to provide sounder evidence of the microbiome features that constitute risk factors for these disorders, as well as dietary intervention studies to establish causality.

In relation to the mechanisms of action, it has been proposed that the microbiota could contribute to triggering the chronic-low grade inflammation underlying insulin resistance and type- 2 diabetes. This hypothesis is supported by the detection of lipopolysaccharide from Gram-negative bacteria in the blood of subjects with metabolic syndrome and type-2 diabetes (40). Moreover, bacterial DNA (mostly belonging to the phylum Proteobacteria) was detected in the blood of subjects before diabetes onset, and was higher in those who had abdominal adiposity (20).

\section{GUT MICROBIOTA AND RISK OF DEVELOPING TYPE-1 DIABETES}

Type- 1 diabetes is an autoimmune disease caused by destruction of insulin-producing $\beta$ cells in the pancreatic islets of Langerhans by immune mediated mechanisms that lead to insulin deficiency in genetically predisposed individuals (41). Although the etiology of the disease is not completely understood, increased intestinal permeability, aberrant immune responses, and intestinal dysbiosis have been proposed as the "perfect storm" that triggers the development of type- 1 diabetes (42). Insufficient regulation of immune attacks on $\beta$ cells, due to genetic and other modifiable factors (e.g., microbiota) is characteristic of disease (43). Increased intestinal permeability has also been described in type-1 diabetes patients (44-46), their relatives (45), and type-1 prediabetic patients $(45,46)$. In this context, it is unclear whether the altered immune status causes intestinal integrity disruption or whether intestinal integrity disruption by an environment trigger (e.g., infections or dysbiosis) causes an abnormal immune response. Possibly both processes are involved.

\section{Mechanisms Behind the Role of Gut Microbiota in Type-1 Diabetes in Animals}

Animal studies support the involvement of intestinal microbiota in the early events that precede and lead to type-1 diabetes, and some suggest that interactions with the host immune system mediate the effects of microbiota in this disorder. Some studies described changes in microbiota composition preceding its onset in diabetic-prone BioBreeding rats (47). Others describe that germ-free mice have increased diabetes incidence or that specific monocolonization of germ-free nonobese diabetic (NOD) mice delays the onset and reduces the incidence of diabetes (48). A number of animal studies also demonstrate that exposure to bacterial antigens or infection in the early neonatal period prevents type-1 diabetes, thereby supporting the notion that microbial immunostimulation may beneficially affect the maturation of the postnatal immune system and protect against type- 1 diabetes (48). The role of the microbiota and its interaction with the innate immune system in type-1 diabetes was demonstrated in a study showing that NOD mice deficient in the innate signaling molecule MyD88 were protected from developing type-1 diabetes (5). This protection was lost when Myd88-/- NOD mice were housed under germ-free conditions, suggesting that the microbiota exerted a protective role. The absence of MyD88 in NOD mice led to changes in microbiota composition, characterized by increases in bacteria of the phylum Bacteroidetes, which could suppress the development of diabetes, presumably through the production of an immunomodulatory compound (5). A more recent study demonstrated that the protective functions of the microbiota against type-1 diabetes development could be transferred (7). Gut bacteria from MyD88-deficient mice, administered over a 3-wk period, stably altered the composition of the gut microbiome (increasing Lachnospiraceae and Clostridiaceae and decreasing Lactobacillaceae), reducing insulitis and delaying the onset of diabetes. This affected the mucosal immune system, increasing the concentration of IgA and transforming growth factor beta in the lumen and of $\mathrm{CD}^{+} \mathrm{CD}^{+} 03^{+}$and $\mathrm{CD} 8 \alpha \beta \mathrm{T}$ cells in the lamina propria of the large intestine, possibly delaying the development of autoimmune diabetes. Administration of the probiotic product VSL\#3 (a combination of strains of the genera Bifidobacterium, Lactobacillus, and Streptococcus) to NOD mice also attenuated destructive insulitis, and preserved beta cells. This was presumably due to the induction of interleukin-10 producing lymphocytes that recirculated from the gut to pancreatic islet (49). Recently, a gluten-free diet has been demonstrated to reduce the diabetic outcomes in NOD mice due to changes in the mouse microbiome (increased Bacteroides and Akkermansia numbers), via stimulation of a higher percentage of $\mathrm{CD} 4{ }^{+} \mathrm{CD} 25^{+} \mathrm{Foxp} 3$ regulatory cells (50).

\section{Evidence Supporting a Role of Gut Microbiota in Type-1 Diabetes in Humans}

Studies reporting associations between shifts in gut microbiota composition and function and type- 1 diabetes in humans are summarized in Table 2. Although results on the gut microbiota components and functions contributing to type- 1 diabetes are not fully consistent, butyrate producing bacteria and bifidobacteria seem to be protective, whereas Proteobacteria/ enterobacteria seem to constitute risk factors for disease. From a functional point of view, the protective role of the commensal intestinal microbiota against this disorder could be mediated by the fact the microbiota and its metabolites (SCFAs) can strengthen mucosal integrity, modulate gut hormone production improving glucose metabolism, and reduce inflammation and prevalence of potential proinflammatory bacteria (e.g., enterobacteria) $(51,53)$.

Other environmental factors with an impact on the gut microbiota and immune system have also been linked to the risk of developing type-1 diabetes, including viral infections, type of delivery (natural birth or caesarian), and infant feeding practices (42). A meta-analysis showed that birth by caesarean section increased the risk of developing the disease by $20 \%$ regardless of the gestational age, weight, maternal age, breastfeeding practices, and maternal diabetes (55). The lack of breastfeeding has also been associated with type-1 diabetes 


\section{\begin{tabular}{l|l} 
Review & Sanz et al.
\end{tabular}}

Table 2. Changes in the intestinal microbiota associated with type-1 diabetes (T1D) in humans

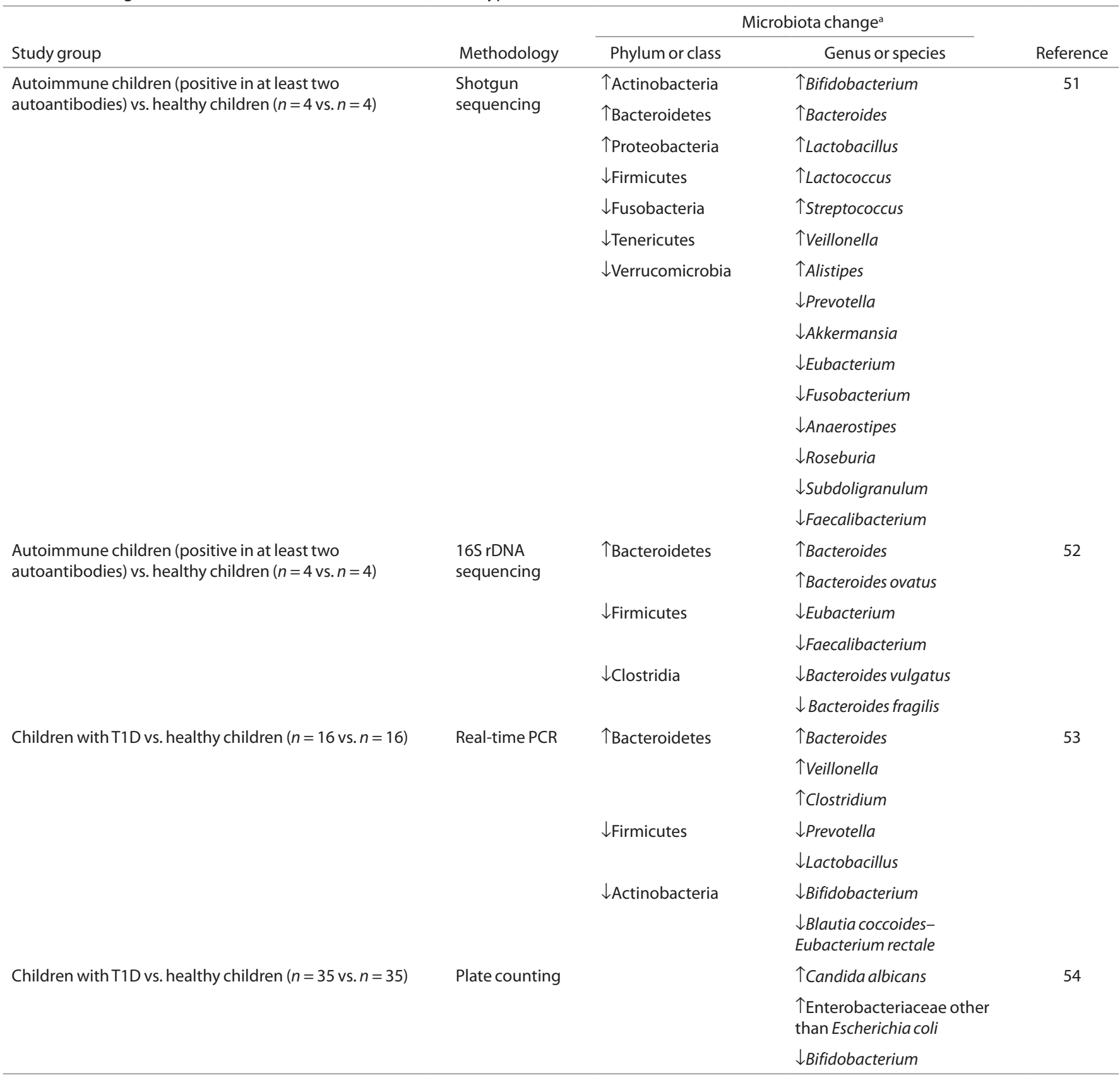

aArrows indicate increases or decreases of each bacterial group in the disease subject group compared with the control group (healthy subjects).

(reviewed in Pereira et al., 2014 (56)). Thus, it is plausible that differences in the acquisition and evolution of the newborn microbiota in caesarian vs. vaginally born infants, and in formula vs. breastfed infants, may also influence the risk of developing type-1 diabetes (57). The association of these factors with other autoimmune disorders such as celiac disease (CD) has been reported (58). Type-1 diabetes and CD are autoimmune disorders with common genetic determinants (HLA-DQ) that frequently coexist. In fact, CD affects at least $10 \%$ of diabetic patients at some point in their life (59). The influence of the HLA-DQ2/8 genotype on the intestinal microbiota composition of infants at family risk of $\mathrm{CD}$ has been described $(1,60)$. However, the influence of the HLA-DQ genotype on the microbiota in subjects at risk of type- 1 diabetes has not been specifically studied, although they share some genetic determinants of $\mathrm{CD}$.

There are also speculations on the role of infections in the risk of developing type-1 diabetes, although no clear conclusions can be drawn from existing human data. For instance, the reduction in the antibody levels against Helicobacter pylori, cytomegalovirus, Epstein-Barr virus, and Toxoplasma in type-1 diabetic patients supports the "hygiene hypothesis" according to which reduced exposure to microbial stimulus increases the disease risk (61). Infections by two coxsackieviruses (B3 and 


\section{Gut microbiome and metabolic disease}

B6) have also been linked to a reduced risk of type-1 diabetes, which was considered to be a result of immunological crossprotection (62). By contrast, intestinal infections by rotavirus $(63)$ and other enterovirus $(62,64)$ have been associated with the onset or with the risk of disease, suggesting that they may contribute to triggering type-1 diabetes. In this vein, an observational study involving type- 1 diabetics under $15 \mathrm{y}$ of age described that perinatal infections were associated with an increased risk in the autoimmune disorder; conversely, attendance to preschool day-care, which promotes microbial exposure, decreased type-1 diabetes risk (65). Overall, the findings suggest that the type of microbial agent, as well as time of exposure, can determine its role in disease risk, although confounding factors could also be a major source of variation in the results.

\section{Conclusions}

Gut microbiota has recently been recognized to play a major role in regulating the metabolic-immune axis, and consequently, changes in gut microbiota related to lifestyle (type of delivery, hygienic measures, diet, etc.) may contribute to the increased incidence of autoimmune and metabolic diseases in developed countries. Type- 1 and type- 2 diabetes represent two examples of diseases mediated by interactions between the gut microbiota and the immune system with subsequent development of specific organ autoimmunity and/or metabolic dysfunction. Knowledge of the role gut microbiota plays in these two diseases could be used to develop intervention strategies to prevent and/or treat imbalances that lead to the inflammation preceding overt manifestations of autoimmune and metabolic disorders. The specific microbiome components promoting or protecting against these disorders and the possible molecular targets for intervention are still under debate. Nonetheless, mechanistic insights from human and animal studies point to the protective role of the intestinal bacteria that produce butyrate, which may strengthen the gut barrier function, and that induce immunoregulatory mechanisms, commonly altered in both type- 2 and type- 1 diabetes. Nevertheless, well-controlled prospective human studies are still necessary to gain a better understanding of the contribution of specific gut microbiota and its response to environmental factors. Such information could be used to identify effective preventive strategies targeting specific component of the gut ecosystem.

\section{STATEMENT OF FINANCIAL SUPPORT}

This review was supported by public grants AGL2011-25169 from the Spanish Ministry of Economy and Competitiveness (MINECO; Spain) and EC Project No. 613979 (MyNew Gut) from the 7th Framework Program. The scholarships to M. Olivares from CSIC (Spain) and to A. Moya-Pérez from the Ministry of Economy and Competitiveness (MINECO; Spain) are fully acknowledged.

Disclosure: There are no disclosures related to financial ties to products in the study or potential/perceived conflicts of interest.

\section{REFERENCES}

1. Olivares M, Neef A, Castillejo G, et al. The HLA-DQ2 genotype selects for early intestinal microbiota composition in infants at high risk of developing celiac disease. Gut 2014 (doi:10.1136/gutjnl-2014-306931).
2. Salonen A, Lahti L, Salojarvi J, et al. Impact of diet and individual variation on intestinal microbiota composition and fermentation products in obese men. ISME J 2014 (doi:10.1038/ismej.2014.63).

3. Maslowski KM, Mackay CR. Diet, gut microbiota and immune responses. Nat Immunol 2011;12:5-9.

4. Sela DA, Mills DA. The marriage of nutrigenomics with the microbiome: the case of infant-associated bifidobacteria and milk. Am J Clin Nutr 2014;99:697S-703S.

5. Wen L, Ley RE, Volchkov PY, et al. Innate immunity and intestinal microbiota in the development of Type 1 diabetes. Nature 2008;455:1109-13.

6. Vijay-Kumar M, Aitken JD, Carvalho FA, et al. Metabolic syndrome and altered gut microbiota in mice lacking Toll-like receptor 5. Science 2010;328:228-31.

7. Peng J, Narasimhan S, Marchesi JR, Benson A, Wong FS, Wen L. Long term effect of gut microbiota transfer on diabetes development. J Autoimmun 2014;53:85-94.

8. Musso G, Gambino R, Cassader M. Obesity, diabetes, and gut microbiota: the hygiene hypothesis expanded? Diabetes Care 2010;33:2277-84.

9. Sanz Y, Moya-Pérez A. Microbiota, inflammation and obesity. In: Lyte Mark, Cryan John F, eds. Microbial Endocrinology: The Microbiota-Gut-Brain Axis in Health and Disease. New York: Springer, 2014:291-317.

10. Caesar R, Reigstad CS, Bäckhed HK, et al. Gut-derived lipopolysaccharide augments adipose macrophage accumulation but is not essential for impaired glucose or insulin tolerance in mice. Gut 2012;61:1701-7.

11. Serino M, Luche E, Gres S, et al. Metabolic adaptation to a high-fat diet is associated with a change in the gut microbiota. Gut 2012;61:543-53.

12. Bäckhed F, Manchester JK, Semenkovich CF, Gordon JI. Mechanisms underlying the resistance to diet-induced obesity in germ-free mice. Proc Natl Acad Sci USA 2007;104:979-84.

13. Moore AM, Munck C, Sommer MO, Dantas G. Functional metagenomic investigations of the human intestinal microbiota. Front Microbiol 2011;2:188.

14. Rey FE, Faith JJ, Bain J, et al. Dissecting the in vivo metabolic potential of two human gut acetogens. J Biol Chem 2010;285:22082-90.

15. Cani PD, Possemiers S, Van de Wiele T, et al. Changes in gut microbiota control inflammation in obese mice through a mechanism involving GLP2-driven improvement of gut permeability. Gut 2009;58:1091-103.

16. Fernandes CA, Fievez L, Neyrinck AM, Delzenne NM, Bureau F, Vanbever R. Sirtuin inhibition attenuates the production of inflammatory cytokines in lipopolysaccharide-stimulated macrophages. Biochem Biophys Res Commun 2012;420:857-61.

17. Caricilli AM, Picardi PK, de Abreu LL, et al. Gut microbiota is a key modulator of insulin resistance in TLR 2 knockout mice. PLoS Biol 2011;9:e1001212.

18. Kim KA, Gu W, Lee IA, Joh EH, Kim DH. High fat diet-induced gut microbiota exacerbates inflammation and obesity in mice via the TLR4 signaling pathway. PLoS One 2012;7:e47713.

19. Devkota S, Wang Y, Musch MW, et al. Dietary-fat-induced taurocholic acid promotes pathobiont expansion and colitis in I110-/- mice. Nature 2012;487:104-8.

20. Amar J, Serino M, Lange $C$, et al. Involvement of tissue bacteria in the onset of diabetes in humans: evidence for a concept. Diabetologia 2011;54:3055-61.

21. Cano PG, Santacruz A, Trejo FM, Sanz Y. Bifidobacterium CECT 7765 improves metabolic and immunological alterations associated with obesity in high-fat diet-fed mice. Obesity 2013;21:2310-2122.

22. Sanz Y, Rastmanesh R, Agostoni C. Understanding the role of gut microbes and probiotics in obesity: how far are we? Pharmacol Res 2013;69:144-55.

23. Verdam FJ, Fuentes S, de Jonge C, et al. Human intestinal microbiota composition is associated with local and systemic inflammation in obesity. Obesity 2013;21:E607-15.

24. Le Chatelier E, Nielsen T, Qin J, et al. Richness of human gut microbiome correlates with metabolic markers. Nature 2013;500:541-6.

25. Jumpertz R, Le DS, Turnbaugh PJ, et al. Energy-balance studies reveal associations between gut microbes, caloric load, and nutrient absorption in humans. Am J Clin Nutr 2011;94:58-65. 
26. Santacruz A, Marcos A, Warnberg J, et al. Interplay between weight loss and gut microbiota composition in overweight adolescents. Obesity 2009;17:1906-15.

27. Cotillard A, Kennedy SP, Kong LC, et al. Dietary intervention impact on gut microbial gene richness. Nature 2013;500:585-8.

28. Ridaura VK, Faith JJ, Rey FE, et al. Gut microbiota from twins discordant for obesity modulate metabolism in mice. Science 2013;341:1241214.

29. Gauffin Cano P, Santacruz A, Moya Á, Sanz Y. Bacteroides uniformis CECT 7771 ameliorates metabolic and immunological dysfunction in mice with high-fat-diet induced obesity. PLoS One 2012;7:e41079.

30. Kootte RS, Vrieze A, Holleman F, et al. The therapeutic potential of manipulating gut microbiota in obesity and type 2 diabetes mellitus. Diabetes, Obes Metab 2012;14:112-20.

31. Kalliomäki M, Collado MC, Salminen S, Isolauri E. Early differences in fecal microbiota composition in children may predict overweight. Am J Clin Nutr 2008;87:534-8.

32. Bergström A, Skov TH, Bahl MI, et al. Establishment of intestinal microbiota during early life: a longitudinal, explorative study of a large cohort of Danish infants. Appl Environ Microbiol 2014;80:2889-900.

33. Santacruz A, Collado MC, García-Valdés L, et al. Gut microbiota composition is associated with body weight, weight gain and biochemical parameters in pregnant women. Br J Nutr 2010;104:83-92.

34. Karlsson CL, Molin G, Cilio CM, Ahrné S. The pioneer gut microbiota in human neonates vaginally born at term-a pilot study. Pediatr Res 2011;70:282-6.

35. Larsen N, Vogensen FK, van den Berg FW, et al. Gut microbiota in human adults with type 2 diabetes differs from non-diabetic adults. PLoS One 2010;5:e9085.

36. Wu X, Ma C, Han L, et al. Molecular characterisation of the faecal microbiota in patients with type II diabetes. Curr Microbiol 2010;61:69-78.

37. Qin J, Li Y, Cai Z, et al. A metagenome-wide association study of gut microbiota in type 2 diabetes. Nature 2012;490:55-60.

38. Karlsson FH, Tremaroli V, Nookaew I, et al. Gut metagenome in European women with normal, impaired and diabetic glucose control. Nature 2013;498:99-103.

39. Zhang X, Shen D, Fang Z, et al. Human gut microbiota changes reveal the progression of glucose intolerance. PLoS One 2013;8:e71108.39.

40. Pussinen PJ, Havulinna AS, Lehto M, Sundvall J, Salomaa V. Endotoxemia is associated with an increased risk of incident diabetes. Diabetes Care 2011;34:392-7.

41. American Diabetes Association. Diagnosis and classification of diabetes mellitus. Diabetes Care 2014;37:Suppl 1:S81-90.

42. Vaarala O, Atkinson MA, Neu J. The "perfect storm" for type 1 diabetes: the complex interplay between intestinal microbiota, gut permeability, and mucosal immunity. Diabetes 2008;57:2555-62.

43. Roep BO, Tree TI. Immune modulation in humans: implications for type 1 diabetes mellitus. Nat Rev Endocrinol 2014;10:229-42.

44. Secondulfo M, Iafusco D, Carratu R, et al. Ultrastructural mucosal alterations and increased intestinal permeability in non-celiac, type I diabetic patients. Dig Liver Dis 2004;36:35-45.

45. Sapone A, de Magistris L, Pietzak M, et al. Zonulin upregulation is associated with increased gut permeability in subjects with type 1 diabetes and their relatives. Diabetes 2006;55:1443-9.

46. Bosi E, Molteni L, Radaelli MG, et al. Increased intestinal permeability precedes clinical onset of type 1 diabetes. Diabetologia 2006;49:2824-7.

47. Brugman S, Klatter FA, Visser JT, et al. Antibiotic treatment partially protects against type 1 diabetes in the Bio-Breeding diabetes-prone rat. Is the gut flora involved in the development of type 1 diabetes? Diabetologia 2006;49:2105-8.

48. King C, Sarvetnick N. The incidence of type-1 diabetes in NOD mice is modulated by restricted flora not germ-free conditions. PLoS One 2011;6:e17049.

49. Calcinaro F, Dionisi S, Marinaro M, et al. Oral probiotic administration induces interleukin-10 production and prevents spontaneous autoimmune diabetes in the non-obese diabetic mouse. Diabetologia 2005;48:1565-75.

50. Marietta EV, Gomez AM, Yeoman C, et al. Low incidence of spontaneous type 1 diabetes in non-obese diabetic mice raised on gluten-free diets is associated with changes in the intestinal microbiome. PLoS One 2013;8:e78687.

51. Brown CT, Davis-Richardson AG, Giongo A, et al. Gut microbiome metagenomics analysis suggests a functional model for the development of autoimmunity for type 1 diabetes. PLoS One 2011;6:e25792.

52. Giongo A, Gano KA, Crabb DB, et al. Toward defining the autoimmune microbiome for type 1 diabetes. ISME J 2011;5:82-91.

53. Murri M, Leiva I, Gomez-Zumaquero JM, et al. Gut microbiota in children with type 1 diabetes differs from that in healthy children: a case-control study. BMC Med 2013;11:46.

54. Soyucen E, Gulcan A, Aktuglu-Zeybek AC, Onal H, Kiykim E, Aydin A. Differences in the gut microbiota of healthy children and those with type 1 diabetes. Pediatr Int 2014;56:336-43.

55. Cardwell CR, Stene LC, Joner G, et al. Caesarean section is associated with an increased risk of childhood-onset type 1 diabetes mellitus: a meta-analysis of observational studies. Diabetologia 2008;51:726-35.

56. Pereira PF, Alfenas Rde C, Araújo RM. Does breastfeeding influence the risk of developing diabetes mellitus in children? A review of current evidence. J Pediatr (Rio J) 2014;90:7-15.

57. Dominguez-Bello MG, Costello EK, Contreras M, et al. Delivery mode shapes the acquisition and structure of the initial microbiota across multiple body habitats in newborns. Proc Natl Acad Sci USA 2010;107:11971-5.

58. Decker E, Engelmann G, Findeisen A, et al. Cesarean delivery is associated with celiac disease but not inflammatory bowel disease in children. Pediatrics 2010;125:e1433-40.

59. Rewers M, Liu E, Simmons J, Redondo MJ, Hoffenberg EJ. Celiac disease associated with type 1 diabetes mellitus. Endocrinol Metab Clin North Am 2004;33:197-214, xi.

60. Palma GD, Capilla A, Nova E, et al. Influence of milk-feeding type and genetic risk of developing coeliac disease on intestinal microbiota of infants: the PROFICEL study. PLoS One 2012;7:e30791.

61. Krause I, Anaya JM, Fraser A, et al. Anti-infectious antibodies and autoimmune-associated autoantibodies in patients with type I diabetes mellitus and their close family members. Ann NY Acad Sci 2009;1173:633-9.

62. Laitinen OH, Honkanen H, Pakkanen O, et al. Coxsackievirus B1 is associated with induction of $\beta$-cell autoimmunity that portends type 1 diabetes. Diabetes 2014;63:446-55.

63. Honeyman MC, Coulson BS, Stone NL, et al. Association between rotavirus infection and pancreatic islet autoimmunity in children at risk of developing type 1 diabetes. Diabetes 2000;49:1319-24.

64. Lönnrot M, Korpela K, Knip M, et al. Enterovirus infection as a risk factor for beta-cell autoimmunity in a prospectively observed birth cohort: the Finnish Diabetes Prediction and Prevention Study. Diabetes 2000;49:1314-8.

65. Levy-Marchal C, Patterson CC, Green A, EURODIAB ACE Study Group. Europe and Diabetes. Geographical variation of presentation at diagnosis of type I diabetes in children: the EURODIAB study. European and Dibetes. Diabetologia 2001;44:Suppl 3:B75-80. 